\title{
World Association for the Advancement of Veterinary Parasitology (W.A.A.V.P.) Guideline: Anthelmintic combination products targeting nematode infections of ruminants and horses
}

\author{
Timothy G. Geary ${ }^{\mathrm{a}, *}$, Barry C. Hosking ${ }^{\mathrm{b}}$, Philip J. Skuce ${ }^{\mathrm{c}}$, \\ Georg von Samson-Himmelstjerna ${ }^{\mathrm{d}}$, Steven Maeder ${ }^{\mathrm{e}}$, Peter Holdsworth ${ }^{\mathrm{f}}$, \\ William Pomroy ${ }^{g}$, Jozef Vercruysse ${ }^{\mathrm{h}}$ \\ a Institute of Parasitology, McGill University, Ste-Anne-de-Bellevue, QC, Canada \\ b Yarrandoo RED Centre, Novartis Animal Health Australasia Pty Limited, Kemps Creek, NSW, Australia \\ c Moredun Research Institute, Penicuik, Midlothian, Scotland, United Kingdom \\ d Institute for Parasitology and Tropical Veterinary Medicine, Freie Universität Berlin, Berlin, Germany \\ e Veterinary Medicine Research \& Development, Pfizer Animal Health, Parkville, VIC, Australia \\ f Animal Health Alliance Limited, Canberra, ACT, Australia \\ $\mathrm{g}$ Institute of Veterinary, Animal and Biomedical Sciences, Massey University, Palmerston North, New Zealand \\ h Department of Virology, Parasitology and Immunology, Faculty of Veterinary Medicine, Ghent University, Merelbeke, Belgium
}

\section{A R T I C L E I N F O}

\section{Keywords:}

Efficacy

Nematodes

Cattle

Sheep

Goats

Horses

Worm control

Anthelmintic resistance

Guidelines

\begin{abstract}
A B S T R A C T
Increasing threats from anthelmintic resistant nematode populations warrant and motivate a reappraisal of chemotherapeutic strategies for nematode control in ruminant livestock and horses. The objective of this paper is to present a guideline for the evaluation of products containing two or more constituent anthelmintic actives in a single dosage form for the treatment of nematode infections in these animals. At present, regulatory policies on the approval of such products vary across jurisdictions, and this World Association for the Advancement of Veterinary Parasitology (W.A.A.V.P.) guideline should enable the harmonization of the requirements. This guideline makes clear recommendations on the minimal standards needed, but stresses that registration dossiers for combination anthelmintic products submitted for approval must conform to the standards and practices already established in existing guidelines for anthelmintics.
\end{abstract}

\section{Introduction}

Economic pressure to obtain optimal performance in ruminant livestock production has guided the use of anthelmintics for many decades. Although there are a number of different approaches to the control of helminth parasites in ruminant livestock and horses, current practice typically relies on the use of highly efficacious broad-spectrum anthelmintics. Though unsustainable with regard to selection for anthelmintic resistance (AR),

\footnotetext{
* Corresponding author. Tel.: +1 514398 7612; fax: +1 5143987857 .

E-mail address: timothy.g.geary@mcgill.ca (T.G. Geary).
}

routine treatment of the entire herd or flock rather than selective treatment of individuals (Corwin, 1997; Charlier et al., 2009) has become commonplace, encouraged by data showing that chemotherapeutic control of even subclinical helminth parasitism can generate a return on investment through gains in production (i.e., meat, milk, wool and reproduction). This strategy, based on cost-benefit analysis in ruminants, has become so ingrained that it can be quite difficult to persuade producers to adopt practices that may lead to reduced short-term income to protect the long-term utility of an anthelmintic product.

When first introduced, the broad-spectrum anthelmintics, including benzimidazoles (BZ), nicotinic acetylcholine receptor (nAChR) agonists (e.g., levamisole, 
LEV) and the macrocyclic lactones (ML), were highly efficacious. However, intensive use has selected drugresistant parasite populations globally in many animal species within a decade of the introduction of every anthelmintic class, and AR is now a major global problem in small ruminants (Kaplan, 2004; Jabbar et al., 2006; Waghorn et al., 2006; Kaplan and Vidyashankar, 2012) and horses (Molento et al., 2012; Reinemeyer, 2012), and is emerging in cattle (El-Abdellati et al., 2010; Sutherland and Leathwick, 2011; Kaplan and Vidyashankar, 2012). For the purposes of this guideline, AR may be simply defined as a heritable change in susceptibility to an anthelmintic in a population of parasitic nematodes such that a dose which normally provides $\geq 95 \%$ clearance of adult worms provides $\leq 80 \%$ clearance. Anthelmintic resistance is arguably the greatest threat to the sustainable control of helminthoses in the short- to medium-term and the problem is compounded by the fact that many of these parasite populations are resistant to more than one class of anthelmintic (van Wyk et al., 1997; Love et al., 2003; Anziani et al., 2004; Wrigley et al., 2006; Sargison et al., 2007; Sutherland et al., 2008; Gasbarre et al., 2009a,b; Cezar et al., 2010; Baker et al., 2012; Molento et al., 2012; Reinemeyer, 2012). Despite the scarcity of well-structured surveys, it is generally accepted that the prevalence of AR is increasing globally in the ruminant livestock industries and in horses.

\section{Combining anthelmintics: current situation}

Drug combinations are commonly used for chemotherapeutic indications in human medicine, including cancer as well as viral, bacterial and protozoan parasitic infections (White, 1999; Miles et al., 2002; Anonymous, 2004; Harrigan et al., 2005; Lane, 2006; Airley, 2009; World Health Organization, 2009; Bang, 2010; Hastings, 2011). The principle that combinations of chemotherapeutic agents benefit patients by maintaining drug efficacy in the presence of resistance has been repeatedly demonstrated in this context for diverse pathogens and builds on knowledge gained from insecticide, pesticide and herbicide use (Wood and Mani, 1981; Curtis, 1985; Mani, 1985; Comins, 1986; Diggle et al., 2003). Currently, combinations of two or more anthelmintics are primarily being used to manage AR in ruminants (i.e., by delaying the emergence and spread of resistance, and/or controlling parasite populations with existing resistance), and to enlarge/expand the spectrum of efficacy.

The increasing problem of AR resulted in the development, approval and widespread adoption of combinations of two or more anthelmintic constituent actives in Australia and New Zealand, where serious problems of AR in nematodes of small ruminants (both countries) and cattle (primarily New Zealand) exist (Besier, 2003; Waghorn et al., 2006; Sutherland and Leathwick, 2011). The global spread of AR in sheep, goats and horses, coupled with the emerging problem of AR in cattle, mandates the development of new products and the implementation of more sustainable application strategies to ensure adequate parasite control in the future. Such strategies may include the use of anthelmintic combinations to forestall productivity losses due to AR, along with the discovery and development of novel anthelmintics. New drugs may well exhibit a reduced spectrum compared to currently available drugs, but could provide effective parasite control in regimens compatible with current (localized) management practices if developed as combination products. This situation supports the contention that current anthelmintic combination guidelines are inadequate and a consensus is urgently needed to motivate and facilitate new product development and regulatory approval.

Anthelmintic combination products incorporating two or more constituent actives are also used to expand the spectrum of efficacy against nematode parasites. For example, a new anthelmintic, derquantel (spiroindole class), has recently been approved for use in some countries as a combination anthelmintic product with abamectin (ML class). By adding abamectin to derquantel, the spectrum of parasite species against which this combination exhibits $\geq 95 \%$ efficacy is significantly increased (Little et al., 2010, 2011).

There is precedence for licensing anthelmintic combination products incorporating two or more constituent actives to expand efficacy against helminth parasites to include organisms in more than one phylum (i.e., nematodes plus trematodes or cestodes). These combination products are often developed based more on a combination of commercial interest and convenience for the end-user than on rigorous considerations of differences in the epidemiology of the disparate helminth targets of the constituent actives. Combinations of a broadspectrum anthelmintic with a flukicide (e.g., clorsulon or triclabendazole) or cestocide (e.g., praziquantel) are available world-wide, for instance, but the appropriate timing of fluke treatment may be an inappropriate time for nematode treatment. While it is legitimate to be concerned that anthelmintic combination products may promote indiscriminate or over-use of the product, the commercial reality is that veterinary pharmaceutical companies develop these products in response to producer demands and seek regulatory approval on this basis. Moreover, they mitigate the risk that end-users will dose animals with self-prepared 'cocktail' mixtures that could contain incompatible components, including excipients, or that may be dosed at incorrect rates. This kind of self-compounded combination of two or more narrow- or broad-spectrum anthelmintics, even though legal in some countries under veterinary supervision, is much less desirable than a combination anthelmintic product that has gone through the regulatory approval process, which will ensure that all the components of the product, including excipients and constituent actives, meet requirements for stability, safety (target animal, user, consumer and environment) and efficacy.

Current problems for the approval of anthelmintic combination products include the lack of universally accepted guidelines for their development; various regulatory agencies employ different approaches and policies for granting approval of combination anthelmintic products for use in ruminant livestock and horses. This situation may result in poor management practices caused by the present reluctance to accept anthelmintic combinations in some jurisdictions (e.g., the European Union and the United 
States of America). Since the control of resistant nematodes may be similarly accomplished by separate dosing with multiple constituent actives in individual products, a practice not prevented under existing regulations, it is unlikely that a combination anthelmintic product would incentivize irresponsible use to a greater extent than what already exists with single-active products.

As noted, guidelines governing the approval of combinations of anthelmintic constituent actives in fixed-dose, single dosage form products vary among countries. The driving force for approval in countries in which these products are registered has been the provision of medicines capable of controlling multiple species of drug-resistant nematodes (including populations resistant to one or more classes of anthelmintic), primarily of sheep and more recently cattle, and also to retard the development and spread of AR as a longer-term goal. Control of existing populations of resistant parasites is much easier to demonstrate and serves as the basis for approval in countries in which combination products are allowed. Data are emerging to support the use of combination anthelmintic products to slow the development and spread of AR, but it would be challenging to incorporate data for this indication per se to support regulatory approval. Instead, label requirements for these products include demonstration of high efficacy (e.g., in Australia $>95 \%$, Anonymous, not dated) against a panel of resistant parasite species relevant for the country. This is feasible for sheep parasites, but may be difficult to attain for resistant parasites of cattle, which are less readily available as experimental isolates; the same situation pertains to equine parasites.

\section{Objectives of the anthelmintic combination guideline}

This new guideline relating to combination anthelmintic products containing two or more constituent actives is developed in the context of those used by countries that already approve their use along with the W.A.A.V.P. and VICH guidelines for general efficacy of anthelmintics (Anonymous, 1999a,b, 2000a,c, 2001; Vercruysse et al., 2001, 2002).

The goal is to provide a harmonized guideline for use by veterinary pharmaceutical companies developing dossiers for approval of fixed-dose anthelmintic combination products by veterinary medicines regulators. Thus, the main goal of this guideline is to provide a scientific basis to recommend globally applicable principles governing the approval of fixed-dose combinations of anthelmintic constituent actives. These include combination products designed to (1) cover the desired breadth of spectrum; (2) minimize (delay) the development and spread of resistance to new and existing anthelmintic classes; or (3) overcome existing species-specific resistance profiles. Specifically, it is important to consider the scientific basis for the adoption or prohibition of combination anthelmintics for the ruminant and equine markets, and to promote regulatory policies that enable adequate parasite control while maintaining the highest standards of safety.

The guideline proposes the efficacy data package required to justify the approval of anthelmintic combination products containing two or more constituent actives from different pharmacological anthelmintic classes in fixed-dose, single dosage formulations for use in ruminant livestock (sheep, goats and cattle) and horses. These anthelmintic combination products could contain constituent actives already approved for use in these hosts or new constituent actives not previously approved for sale. The combined constituent actives do not need to exhibit completely overlapping spectra of parasites. Approval should depend on demonstration of the normal standards of product stability, safety, residues and efficacy, particularly against relevant resistant isolates and species of targeted parasites.

Although combinations of drugs that control trematodes rather than nematodes have not been pursued as avidly as combination products for nematodes, their regulatory approval could be justified on similar grounds. Certain trematocides (flukicides) selectively target different stages of the parasite life cycle, and in theory, broader control could be achieved by the use in combination of constituent actives with efficacy against immature and mature stages of Fasciola hepatica, for instance. While considerations similar to those proposed here for regulatory approval of nematocidal combination products could be applied to trematocidal combination products, specific discussion of these latter products will not be provided here.

This guideline does not consist of rigid stipulations, but instead makes recommendations on the minimum studies required (Wood et al., 1995; Vercruysse et al., 2001). Guidelines cannot address all possibilities and each case should be considered on its merits; if alternative approaches are deemed more fitting, a reasoned argument should be discussed with regulatory agencies in the target jurisdiction. Before describing the anthelmintic combination guideline, rationales for the use of anthelmintic combination products and concerns about fixed-dose anthelmintic combination products will be discussed.

\section{Rationales for the use of anthelmintic combination products}

To deny on principle the use of combination products for nematode infections requires some evidence that such infections are fundamentally different from infections with other kinds of pathogens (or from cancer). One exception pertains to the therapeutic coverage required. For viral, bacterial and fungal infections, as well as cancer, the target is $100 \%$ elimination of the pathogen (including tumor cells) in every treated patient. These infections are addressed as individual cases, unlike the population treatment paradigms typically employed in anthelmintic treatment strategies for ruminant livestock (and sometimes horses). Indeed, a key principle for slowing the emergence of AR in parasitic nematodes of ruminant livestock or horses is to obtain the highest possible removal of the parasites in significantly infected animals while ensuring that surviving parasites are diluted into a pool of susceptible, untreated parasites in the local environment as a refugial population (Dobson et al., 2001, 2011b; Bartram et al., 2012). This principle applies to singleconstituent active and combination products containing 
two or more distinct constituent actives (e.g., anthelmintics from different chemical classes with distinct mechanisms of resistance, or at least demonstrably different mechanisms of action). It should be noted that this difference does not contravene the potential therapeutic utility of anthelmintic combination products for providing efficacy in the presence of AR and in delaying the onset and spread of AR if used according to best practices (see Section 5.3).

\subsection{Managing existing resistance}

As noted, combinations of two or more anthelmintic constituent actives have been approved and widely adopted in Australia and New Zealand for use in ruminants. The first formulated combination products contained a BZ and LEV as constituent actives and were introduced into these countries in the early 1980s to control resistant nematodes in sheep (Anderson et al., 1988; McKenna, 1990). A great deal of experience and knowledge pertaining to the use of such products has subsequently been gained. The wide use of anthelmintic combinations, often necessitated by the very high frequency of resistance to one or more available constituent actives when used alone, has revealed no issues of special concern with the routine use of such products. It was to be expected that some multiply-resistant nematode populations (e.g., Love et al., 2003; Wrigley et al., 2006; Sutherland et al., 2008; Baker et al., 2012) would ultimately be found in countries where combinations were used, given the long history of use of their components as single-constituent active anthelmintic products, generating high 'pre-existing' resistance (R)allele frequencies in exposed parasite populations.

\subsection{Delaying anthelmintic resistance}

The role that anthelmintic combination products could play in delaying the emergence of drug-resistant parasites was identified after commercial products of this type were first introduced to control resistant parasites in sheep (Anderson et al., 1988). Mathematical simulation studies demonstrated that the full benefit of combination anthelmintic product therapy would be realized when initial R-allele frequencies were low, and that the likelihood of resistance occurring to a combination anthelmintic product would increase with increasing R-allele frequency to its individual constituent actives (Smith, 1990; Barnes et al., 1995; Leathwick, 2012; Bartram et al., 2012). This concept was later supported by an empirical study in New Zealand (Leathwick et al., 2012). Fixed-dose commercial anthelmintic combination products contain as many as four anthelmintic constituent actives with different mechanisms of action. Experience with such products for the control of multiple-resistant isolates of several nematode species has not uncovered undue toxicity or safety concerns in the veterinary setting. While resistance to a 4-way anthelmintic combination product (BZ, LEV, abamectin, closantel) has been reported in Haemonchus contortus (Baker et al., 2012), this observation serves only to confirm the benefit of commencing use of combination anthelmintic products when R-allele frequencies are low.
Theoretical considerations of the value of combination anthelmintic products have recently been discussed (Leathwick et al., 2009; Bartram et al., 2012), and the benefits of such products were further demonstrated in a simulation study using an Australian sheep model with multiple parasite species. A key outcome from this modeling was that a (theoretical) 4-way combination of BZ-LEV-abamectin-monepantel (aminoacetonitrile derivative class) was the best option for delaying the development of AR while achieving effective nematode control despite the presence of resistance to the first three drugs (Dobson et al., 2011a,b).

Importantly, experimental and modeling data suggest that the development of resistance to a new anthelmintic will not be accelerated (and in most circumstances it will be delayed) by its inclusion in a combination product (Dobson et al., 2011a,b; Leathwick, 2012; Leathwick et al., 2012). Indeed, this is a driving force for combination chemotherapy of HIV, tuberculosis and malaria in humans (see above) and may be considered one of the key advantages of anthelmintic combinations in geographic locations where AR in cattle parasites is not already intensely distributed (i.e., Europe, North America).

\subsection{Specific targeting of dose-limiting species}

The dose-limiting parasite species will generally be identified during dose determination studies that identify the dose providing $\geq 90 \%$ efficacy. Lower doses will show efficacy $<90 \%$ for the dose-limiting species even though they will adequately treat other parasites (i.e., efficacy $\geq 90 \%$ ) (Vercruysse et al., 2001).

Dose-limiting species can be vulnerable to selection for AR and at least on some occasions have been the first to reveal resistance, as is clearly evidenced with the MLs and cattle parasites. Current AR in cattle parasites is primarily due to isolates of Cooperia spp. (Sutherland and Leathwick, 2011), which are the dose-limiting parasites for this drug class (Vercruysse and Rew, 2002). Incorporating a second anthelmintic constituent active with a ML in a fixed-dose anthelmintic combination product could address concerns around control of these parasites and significantly delay the spread and further selection of resistant Cooperia spp. populations.

\subsection{Maximizing breadth of spectrum}

Two novel drugs with complementary nematode spectra that are separately inadequate for livestock parasite control could be combined in a fixed-dose product to provide therapeutically useful activity. An example in companion animals is the combination of febantel with pyrantel pamoate or oxantel (plus praziquantel), which provides high efficacy against the important gastrointestinal nematode species in a single dose, whereas the single agents require multiple doses for similar results when used alone. There is no apparent disadvantage to this kind of combination compared to a novel single agent product with an equivalent overall spectrum of action, as long as 
safety and residue concerns (if used in food/fiber production species) are adequately addressed.

\section{Concerns about fixed-dose anthelmintic combination products}

A primary principle of infectious disease chemotherapy is to identify the pathogen in order to choose the most appropriate agent and treatment regime. In practice, this principle is often ignored, given the costs associated with diagnostic tests and procedures, and the delay in treatment encountered as the diagnosis is awaited. The availability of truly broad-spectrum antibiotics and anthelmintics has radically changed the expectations of patients, physicians, veterinarians and livestock producers, essentially bypassing the requirement for confirmation of the pre-treatment diagnosis.

Ideally, the species of parasitic nematodes present in a flock or herd would be identified, along with susceptibility testing to determine which class(es) of anthelmintic(s) should be administered. However, this strategy is rarely adopted by commercial operations, as it runs counter to the perception that schemes based on enhanced diagnostics for case management rather than herd or flock treatment add labor costs and reduce convenience. Importantly, animal welfare considerations demand prompt treatment of any animal that is ill due to parasitism. Since the drugresistance status of parasites on farms is rarely determined prior to choosing a treatment (Lawrence et al., 2007; Dobson et al., 2011a; Morgan et al., 2012), an approach of using single-constituent active products for strategic dosing to account for the species and AR status present at the time is not likely to be practical or sustainable even in smaller operations. This will be exacerbated as populations of multiply-resistant parasite populations spread, requiring multiple treatments with different products in separate dosage forms to maintain control. In such situations, fixed-dose anthelmintic combination products, rather than administration of multiple doses of a number of single-constituent active products, would reduce both animal stress and labor costs.

Three primary areas of concern (discussed in the following sections) are apparent with fixed-dose commercial anthelmintic combination products, viz. drug-drug interactions (safety and efficacy implications of pharmacokinetics and pharmacodynamics), common mechanisms of resistance and best-practice management to ensure appropriate use for sustainability of parasite control with the products.

\subsection{Drug-drug interactions}

Safety concerns about fixed-dose anthelmintic combination products are centered on the potential pharmacokinetic and/or pharmacodynamic interactions that may occur between the constituent actives or the excipients used (Alvarez et al., 2008; Entrocasso et al., 2008; Suarez et al., 2009; Lanusse, 2010), and the subsequent complications these interactions could cause for efficacy, residues, target animal safety and environmental safety. However, while approval of a product containing different nematocidal constituent actives in a single dosage is not permitted by some regulatory agencies, no regulations are apparent that prevent the concurrent administration of two or more different registered anthelmintic constituent actives to ruminants or horses at the owner's discretion, providing the products are administered in separate dosage forms. Products containing fixed-dose combinations of multiple anthelmintic constituent actives have been approved and are in wide use in some countries, but the authors are not aware of reports of pharmacokinetic, toxicokinetic or pharmacodynamic interactions associated with these products in ruminant livestock. Nonetheless, the potential for such interactions of individual constituent actives in any combination anthelmintic product should be addressed in each dossier for submission (for an example, see Cromie et al., 2006) (see Section 6).

Combination chemotherapy products often are based on using drugs with similar pharmacokinetic profiles on the grounds that matching elimination curves will protect each of the components from the selection of resistant pathogen populations by maintaining consistent simultaneous exposure. However, concerns about matching half-lives of elimination to minimize exposure to suboptimal concentrations of single constituent actives or their bioactive metabolites at the tail of the elimination curve may be more relevant for synergistic combinations. While synergistic combinations have special value in chemotherapy, and provide an adequate justification for a combination product, additive effects, at the least, are to be expected and also justify a combination anthelmintic product (in contrast, an antagonistic interaction in which the efficacy of the combination of constituent actives is lower than would be predicted by their efficacies when used alone may preclude regulatory approval; Fuhr, 2007). It should not be a requirement for additive combinations that the entirety of the pharmacokinetic profiles of the constituent actives are highly similar; however, adequate overlap of the time-to-kill curve for each agent must be observed to ensure that they are present simultaneously in sufficient concentrations for sufficient duration to attain co-incident lethal exposures. This will subject the parasites to independent chemotherapeutic pressures that will eliminate or reduce the survival of individual worms with R-alleles to only one of the constituent actives in the combination product. It will not protect the longer-duration component from selecting for resistance during a period of suboptimal concentrations at the tail of the elimination curve, but this situation is not different than what would be experienced if that constituent active is used alone in a product. Deviation from this pattern can be tolerated if supported by evidence that differences in the pharmacokinetic profiles of the constituent actives still subject the parasites to the additive effects needed to avoid independent and sequential selection for resistance.

\subsection{Common mechanisms of resistance}

An anthelmintic combination product is only appropriate if its constituent actives do not share the same mechanism of resistance (noting this is different from mechanism of action). Most experience suggests that anthelmintics from the various pharmacological classes do 
not exhibit common mechanisms of resistance, although these mechanisms remain poorly understood; and there is no experimental or confirmed field evidence that developing resistance to one class predisposes to the development of resistance in another class. Mottier and Prichard (2008) have questioned the use of anthelmintic combination products containing a $\mathrm{BZ}$ and a ML on the basis that repeated exposure of $H$. contortus to ML anthelmintics promoted allelic changes in the $\beta$-tubulin isotope 1 gene, the key locus involved in the mechanism of BZ resistance. This observation may have implications for the use of anthelmintic combination products containing constituent actives in these classes, but it is not clear that it represents true cross-resistance (Leathwick et al., 2009). Leathwick et al. (2009) further point out that, while Mottier and Prichard (2008) suggest that MLs may act as modifiers of BZ resistance, no pharmacological evidence has been advanced to show that nematodes resistant to MLs will also be resistant to BZs. Additionally, it is not clear that a limited degree of cross-resistance, should it exist, would be sufficient to nullify the benefits of administering the anthelmintics in combination (Leathwick et al., 2009).

That single-constituent active products from these classes are already used in areas of existing drug resistance suggests that products containing them in combination pose no greater risk of selection for multiple-drug resistance than would be encountered if they were used in rotation or administered concurrently in separate dosage forms.

Similarly, adding a novel constituent active to an anthelmintic combination product that includes existing constituent actives, as opposed to using it alone or in rotation in areas where resistance already exists, should not predispose it to a more rapid selection for evolution of parasite resistance as demonstrated in recent modeling and empirical studies (Dobson et al., 2011a,b; Leathwick, 2012; Leathwick et al., 2012). Proof of efficacy of combinations against existing resistant parasite isolates in dose-determination experiments will alleviate this concern to some extent, although only field use will reliably reveal if selection of a resistance mechanism that crosses anthelmintic classes can occur in nematode populations following the use of a new drug.

\subsection{Best-practice management of combination anthelmintics}

As noted above, few farmers test for AR (Lawrence et al., 2007; Dobson et al., 2011a; Morgan et al., 2012). Under these circumstances, a concern is that fixed-dose combination anthelmintic products could mask the development of resistance. This may be considered a technology transfer or compliance problem that does not change the conclusions from modeling studies that resistance will be substantially delayed by administering anthelmintic combination products in comparison to rotation or sequential use strategies of single-constituent active products (Smith, 1990; Barnes et al., 1995; Leathwick, 2012). Fixed-dose combination anthelmintic products appear to slow the development of resistance because they afford the highest possible kill of nematodes (Bartram et al., 2012). Parasites that survive one constituent active in the combination are killed by the other constituent active(s); individual parasites that possess two distinct R-alleles, each of which is present in the population at very low frequencies, will initially be very rare.

However, the use of anthelmintic combination products does not eliminate the significant risk for resistance posed by dosing strategies that allow livestock to graze clean (low contamination) pastures after treatment. This practice readily selects for resistant populations as the parasites that survive the treatment become the major source of subsequent contamination on these pastures (LeJambre, 1978; Cawthorne and Whitehead, 1983; Michel, 1985; Taylor and Hunt, 1988, 1989). This concern is obviously more acute if resistance to one of the constituent actives in the combination product is already present and unsuitable treatment regimes are implemented.

The benefits of maintaining a population of nematodes in refugia, as a means of slowing the development of drug resistance, were first advanced by Martin et al. (1981) and should not be underestimated; van Wyk (2001) and Dobson et al. (2001) proposed that refugia could be the most important factor in determining the rate at which AR develops. Full consideration should be given to refugia when designing and implementing nematode control programs. With heightened scientific interest in maintaining adequate refugia as a means of slowing the development of $\mathrm{AR}$, considerable improvements have been made in recent years to our understanding of the concept (Kenyon et al., 2009; Leathwick et al., 2009; Bartram et al., 2012).

For ruminants, the number of animals that should be left untreated to create an adequate refuge of parasites will vary between breed and age (i.e., level of immunity), farm management practices, anthelmintic treatments (which includes consideration of efficacy and AR status), nematode species (including the potential for hypobiotic stages), and geographic region (with an overlying influence of climate on the development and survival of the free-living parasite stages on pasture). Examples of generally accepted strategies to establish adequate refugia include: grazing untreated adults with younger animals that are treated; ensuring that the interval between treatments allows some contamination of pasture with unselected parasites; treating animals several days after moving to relatively worm-free pasture to contaminate the area with unselected nematodes; or leaving a proportion of animals with a group untreated. This mix of factors creates an extremely complex environment in which simulation models can be of more benefit than field experiments (Hosking, 2010; Dobson et al., 2011b). Thus, the propensity for selection of resistant nematode populations through an inadequate population of parasites in refugia is a matter of concern for all anthelmintic products and hence is another technology transfer problem for any new product, whether composed of single or multiple constituent actives. It should be noted that little work has been done on the role of refugia in the development of AR in horses, but it would be conservative to assume that the importance is similar to the situation in ruminants.

In any case, animal health advisors must capture every opportunity to strongly reinforce best-practice management to their clients. The principles of continuing 
education are the same whether producers use singleconstituent active or combination anthelmintic products. This includes testing for AR to identify suitable constituent actives, estimating (however inadequately) nematode burdens and species by fecal egg counts (FEC) and preferably larval culture (or PCR) to determine appropriate treatment regimens, and the management of pasture exposures to reduce the overall parasite challenge in balance with the maintenance of drug-susceptible populations in refugia, which can help slow the development of AR in nematodes (Barger, 1999; Dobson et al., 2001, 2011b; van Wyk, 2001; Baker et al., 2012; Bartram et al., 2012).

\section{Efficacy guideline for regulatory approval/registration of anthelmintic combination products to control nematode infections of ruminant livestock and horses}

The composition of product dossiers for regulatory evaluation will be based on existing requirements already established for single-constituent active products, including dose determination and confirmation studies, field and persistent efficacy studies, and using accepted standards of effectiveness and definition of helminth control claims in multiple species according to W.A.A.V.P. guidelines (Jacobs et al., 1994; Wood et al., 1995; Duncan et al., 2002; Yazwinski et al., 2003; Hennessy et al., 2006) and VICH (Anonymous, 1999a,b, 2000a,c, 2001; Vercruysse et al., 2001, 2002).

Dossiers submitted for the approval of anthelmintic combination products to control nematode infections of ruminant livestock and equines should also include at least the following information: (1) justification for the combination, including evidence that the anthelmintic constituent actives do not share a mechanism of resistance, to the extent that this is known, (2) dose determination data for the constituent actives in the combination (of particular importance if one of the constituent actives has not been previously approved and so data are unavailable for reference), (3) target animal safety and pharmacokinetic data showing non-interference and acceptable safety, (4) dose confirmation including persistent efficacy and efficacy against resistant isolates if claimed in the application, and (5) field efficacy.

\subsection{Justification for the combination}

Before a combination anthelmintic product can be considered, a detailed justification for the combination, including the anticipated benefits, is necessary. Justification of combinations should be clearly based on at least one of the following considerations, with each constituent active of the combination addressed:

- Overcoming lack of efficacy for existing resistant nematode species and preservation of the useful clinical activity of existing anthelmintics.

- Delaying the selection and spread of resistance to new anthelmintic constituent actives.

- Specific targeting of dose-limiting parasite species.
- Proven anthelmintic synergism of the constituent actives.

- Additional parasite species claims.

\subsection{Dose determination data on the anthelmintic} constituent actives in the combination

If the anthelmintic constituent actives to be used in the combination product are already registered in the same formulation and same route of administration used for the combination product, efficacy data will be available and new studies will not be required for dose determination if all constituent actives are used at approved doses (and in the absence of untoward results from studies discussed in Section 6.3). However, proof of efficacy of the fixed-dose combination for the intended indications will be required from dose confirmation and field studies (Sections 6.5 and 6.6). If existing anthelmintics are combined in a formulation in which at least one constituent active has not been approved, dose determination studies should be done; pharmacokinetic studies that demonstrate plasmalevel bioequivalence of the new and previously approved formulation do not necessarily predict efficacy in other compartments, including the gastrointestinal tract.

For new anthelmintic constituent actives, dose determination efficacy data must be provided according to existing W.A.A.V.P. (Wood et al., 1995; Duncan et al., 2002) and VICH (Anonymous, 1999a,b, 2000a,b,c; Vercruysse et al., 2001, 2002) guidelines. If claims of synergy are made, conclusive evidence supporting lowered doses (if used) must be provided in accordance with the relevant guidelines. It should be noted that combination products that contain synergistic constituent actives would not require independent testing of the individual anthelmintics, as the efficacy of the combination product would clearly be dependent on their simultaneous presence. Synergistic combinations should instead be evaluated according to existing regulations for single constituent active products.

\subsection{Target animal safety and pharmacokinetic data showing non-interference and acceptable safety}

Although previous efficacy guidelines did not address issues of target animal safety or pharmacokinetics, even though these fields are required for product approval by regulatory authorities, the unique situation pertaining to anthelmintic combination products requires some consideration.

While reports of drug-drug interactions and enhanced toxicity in ruminant livestock or horses are not apparent for anthelmintic combination products, data justifying the combination in terms of possible interactions at the pharmacokinetic and pharmacodynamic levels, and evidence of acceptable safety will nonetheless need to be provided. Safety studies should be conducted with the minimal number of animals required to demonstrate safety; the availability of data from previous approval dossiers that prove safety of the combination of anthelmintic constituent actives in the same formulation, or another formulation that provides pharmacokinetic bioequivalence, could minimize the requirement for additional studies. In 
each case, approval of all dosage forms and routes of administration should be predicated on regulatory requirements for such products established in the various jurisdictions in which approval is sought. Consultation on these requirements should be sought before such studies commence.

\subsection{Product bioequivalence}

The principle of product bioequivalence for the individual anthelmintic constituent actives in question cannot simply be applied to the fixed-dose combination product, as it could comprise formulation changes to the approved individual anthelmintic constituent actives. Pharmacokinetic data alone cannot be used to justify approval of an anthelmintic combination product, because it is not possible to conclude on that basis that the constituent actives will not show pharmacological antagonism against target parasite species. As noted above, there may be a poor correlation between plasma pharmacokinetics and anthelmintic efficacy for gastrointestinal parasites. Thus, the dossier must include data from dose confirmation and field studies proving the efficacy of the combination product, compared to the individual constituent actives administered alone (Section 6.5).

\subsection{Dose confirmation studies}

The design and analysis of dose confirmation studies for the anthelmintic combination product should be based on the rationale for approval of the combination anthelmintic product as described in Section 4. It should be noted in this context that the goal of delaying the onset of AR is unlikely to be adequately addressed in animal studies. Thus, dose confirmation studies need to be conducted in a location(s) and in such a manner that the chosen rationale(s) is supported by data and meets the requirements of the current guidelines for anthelmintics. At least one dose confirmation study must be conducted with the final formulation to be commercialized and should include the dose-limiting parasite(s) for each anthelmintic used in the combination.

The number, location, design and analysis of these studies should be based on existing guidelines for singleconstituent active products (Section 6.2). To investigate efficacy against adult parasites, naturally infected animals are preferred and infections should include, where possible, known drug-resistant isolates or populations of nematodes; strains resistant to one or more of the anthelmintic constituent actives in the combination should be included if available. The efficacy of each individual constituent active in the combination should be verified against the presumed resistant isolates to validate the inclusion of data from these trials in the dossier (e.g., the resistant populations must be proven to be present). Efficacy against larval stages should be determined using experimental infections of nematodes from drug-resistant isolates (including, if available, isolates obtained within the previous 10 years) to permit claims on the product label. For such purposes, 'resistance' can attributed to a population of a parasite species that exhibits substantial reductions in efficacy (e.g., to $\leq 80 \%$ ) when treated with a dose of the anthelmintic which is historically $\geq 95 \%$ efficacious against that species (based on adequate evidence from worm counts and/or FEC reductions) (Coles et al., 1992; Anonymous, 2003). These isolates should encompass the extent of $A R$ present in the country of interest and should be agreed by the relevant regulatory agency as part of the preliminary discussions over the content of the dossier required for consideration for approval. Efficacy against nematode species that can exist as hypobiotic larvae should be included in the studies if label claims for such parasites are sought. Analyses of parasite data in support of efficacy claims should be based on estimates of adult and larval stage worm counts as specified in the earlier guidelines (Section 6.2).

\subsection{Field efficacy studies}

Field efficacy studies shall be conducted using reduction in FEC (with supporting larval culture or PCR data) after treatment with the final formulation of the combination product to be commercialized to confirm efficacy and safety in the field in accordance with the current guidelines for single constituent active products (Wood et al., 1995; Anonymous, 1999a,b, 2000a,b,c; Vercruysse et al., $2001,2002)$. The number of field studies to be conducted and the number of animals in each study will depend on (1) the animal species and age, (2) the geographic location, (3) local/regional regulatory requirements, and (4) the rationale for the combination product as noted in Section 6.1. The controls should include untreated animals and preferably animals treated with registered anthelmintics with known profiles, including those used in the combination product. 'Local/regional' implies within a country and/or association with a climatic and/or management area. To achieve the desired numbers, it is also acceptable to conduct multi-center studies with sub-studies in each locality/region. The request for additional (or fewer) studies, and/or animals (animal welfare considerations) from local regulatory authorities should be fully justified. The combination product should always be tested in the age range/class/production type of animal intended to be treated as indicated on the proposed product label.

\section{Conclusions}

The goal of this guideline is to provide a scientific basis upon which to recommend globally applicable principles governing the approval of fixed-dose, single dosage form combinations of anthelmintic constituent actives with similar spectra of activity. This guideline must be used in conjunction with existing VICH and W.A.A.V.P. guidelines. The authors have identified no scientific considerations that should prevent approval of properly justified anthelmintic combination products for use in ruminant livestock and horses to control nematode parasites.

\section{Acknowledgments}

The authors gratefully acknowledge and thank Bert Stromberg, Ray Kaplan, Roger Prichard, Andy Forbes, Roger 
Sargent and Jan van Wyk who provided constructive criticisms on the manuscript.

\section{References}

Airley, R., 2009. Cancer Chemotherapy. John Wiley \& Sons, West Sussex, UK, 342 pp.

Alvarez, L., Lifschitz, A., Entrocasso, C., Manazza, J., Mottier, L., Borda, B., Virkel, G., Lanusse, C., 2008. Evaluation of the interaction between ivermectin and albendazole following their combined use in lambs. J. Vet. Pharmacol. Ther. 31, 230-239.

Anderson, N., Martin, P.J., Jarrett, R.G., 1988. Mixtures of anthelmintics: a strategy against resistance. Aust. Vet. J. 65, 62-64.

Anonymous, not dated. Efficacy standards for ruminant anthelmintics in Australia. Australian Pesticides and Veterinary Medicines Authority. http://www.apvma.gov.au/publications/guidelines/docs/ anthelmintics_efficacy.pdf (accessed 11.05.11).

Anonymous, 1999a. Efficacy of anthelmintics: specific recommendations for bovines. VICH Guideline 12. http://www.vichsec. org/pdf/2000/GL12_ST7.pdf (accessed 02.09.11).

Anonymous, 1999b. Efficacy of anthelmintics: specific recommendations for ovines. VICH Guideline 13. http://www.vichsec.org/ pdf/2000/GL13_ST7.pdf (accessed 11.05.11).

Anonymous, 2000a. Efficacy of anthelmintics: general requirements. VICH Guideline 7. http://www.vichsec.org/pdf/Gl07_st7final+corr.pdf (accessed 11.05.11).

Anonymous, 2000b. Good clinical practice. VICH Guideline 9. http://www.vichsec.org/pdf/2000/Gl09_st7.pdf (accessed 11.05.11).

Anonymous, 2000c. Efficacy of anthelmintics: specific recommendations for caprines. http://www.vichsec.org/pdf/2000/GL14_ST7.pdf (accessed 24.10.11).

Anonymous, 2001. Efficacy of anthelmintics: specific recommendations for equines. http://www.vichsec.org/pdf/07_2001/gl15_st7.pdf (last accessed 11.11.11)

Anonymous, 2003. ACVM Registration standard and guideline for efficacy of anthelmintics in cattle, sheep, goats and deer. New Zealand Food Safety Authority. http://www.foodsafety.govt.nz/ elibrary/industry/Acvm_Registration-Chemicals_Medicines.pdf (accessed 11.11.11).

Anonymous, 2004. Roll back malaria. http://rbm.who.int/partnership/ board/meetings/docs/strategy_rev.pdf (accessed 16.09.11).

Anziani, O.S., Suarez, V., Guglielmone, A.A., Warnke, O., Grande, H., Coles, G.C., 2004. Resistance to benzimidazole and macrocyclic lactone anthelmintics in cattle nematodes in Argentina. Vet. Parasitol. 122, 303-306.

Baker, K.E., George, S.D., Stein, P.A., Seewald, W., Rolfe, P.F., Hosking, B.C., 2012. Efficacy of monepantel and anthelmintic combinations against multiple-resistant Haemonchus contortus in sheep, including characterisation of the nematode isolate. Vet. Parasitol. 186, 513-517.

Bang, D., 2010. The management of tuberculosis: epidemiology, resistance and monitoring. Dan. Med. Bull. 57 (11), B4213.

Barger, I.A., 1999. The role of epidemiological knowledge and grazing management for helminth control in small ruminants. Int. J. Parasitol. 29, 41-47.

Barnes, E.H., Dobson, R.J., Barger, I.A., 1995. Worm control and anthelmintic resistance: adventures with a model. Parasitol. Today 11, 56-63.

Bartram, D.J., Leathwick, D.M., Taylor, M.A., Geurden, T., Maeder, S.J., 2012. The role of combination anthelmintic formulations in the sustainable control of sheep nematodes. Vet. Parasitol. 186, 151-158.

Besier, R.B., 2003. Anthelmintic resistance in sheep nematodes in Australia: the need for new approaches. Austral. J. Exp. Agric. 43, 1383-1391.

Cawthorne, R.J.G., Whitehead, J.D., 1983. Isolation of benzimidazole resistant strains of Ostertagia circumcincta from British sheep. Vet. Rec. 112, 274-277.

Cezar, A.S., Toscan, G., Camillo, G., Sangioni, L.A., Ribas, H.O., Vogel, F.S.F., 2010. Multiple resistance of gastrointestinal nematodes to nine different drugs in a sheep flock in southern Brazil. Vet. Parasitol. 173, $157-160$.

Charlier, J., Höglund, J., von Samson-Himmelstjerna, G., Dorny, P., Vercruysse, J., 2009. Gastrointestinal nematode infections in adult dairy cattle: impact on production, diagnosis and control. Vet. Parasitol. 164, 70-79.

Coles, G.C., Bauer, C., Borgsteede, F.H.M., Klei, T.R., Taylor, M.A., Waller, P.J., 1992. World Association for the Advancement of Veterinary Parasitology (W.A.A.V.P.) methods for the detection of anthelmintic resistance in nematodes of veterinary importance. Vet. Parasitol. 44, 35-44.
Comins, H.N., 1986. Tactics for resistance management using multiple pesticides. Agric. Ecol. Environ. 16, 129-148.

Corwin, R.M., 1997. Economics of gastrointestinal parasitism in cattle. Vet. Parasitol. 72, 451-457.

Cromie, L., Ferry, M., Couper, A., Fields, C., Taylor, S.M., 2006. Pharmacokinetics of a novel closantel/ivermectin injection in cattle. J. Vet. Pharmacol. Ther. 29, 205-211.

Curtis, C.F., 1985. Theoretical models of the use of insecticide mixtures for the management of resistance. Bull. Entomol. Res. 75, 259-265.

Diggle, A.J., Neve, P.B., Smith, F.P., 2003. Herbicides used in combination can reduce the probability of herbicide resistance in finite weed populations. Weed Res. 43, 371-382.

Dobson, R.J., Besier, R.B., Barnes, E.H., Love, S.C.J., Vizard, A., Bell, K., Le Jambre, L.F., 2001. Principles of use of macrocyclic lactones to minimise selection for resistance. Aust. Vet. J. 79, 756-761.

Dobson, R.J., Hosking, B.C., Besier, R.B., Love, S., Larsen, J.W.A., Rolfe, P.F., Bailey, J.N., 2011a. Minimising the development of anthelmintic resistance, and optimising the use of the novel anthelmintic monepantel, for the sustainable control of nematode parasites in Australian sheep grazing systems. Aust. Vet. J. 89, 160-166.

Dobson, R.J., Barnes, E.H., Tyrrell, K.L., Hosking, B.C., Larsen, J.W.A., Besier, R.B., Love, S., Rolfe, P.F., Bailey, J.N., 2011b. A multi-species model to assess the impact of refugia on worm control and anthelmintic resistance in sheep grazing systems. Aust. Vet. J. 89, 200-208.

Duncan, J.L., Abbott, E.M., Arundel, J.H., Eysker, M., Klei, T.R., Krecek, R.C., Lyons, E.T., Slocombe, J.O.D., 2002. World Association for the Advancement of Veterinary Parasitology (W.A.A.V.P.) second edition of guidelines for evaluating the efficacy of equine anthelmintics. Vet. Parasitol. 103, 1-18.

El-Abdellati, A., Charlier, J., Geldhof, P., Levecke, B., Demeler, J., von Samson-Himmelstjerna, G., Claerebout, E., Vercruysse, J., 2010. The use of a simplified faecal egg count reduction test for assessing anthelmintic efficacy on Belgian and German cattle farms. Vet. Parasitol. 169, 352-357.

Entrocasso, C., Alvarez, L., Manazza, J., Lifschitz, A., Borda, B., Virkel, G., Mottier, L., Lanusse, C., 2008. Clinical efficacy assessment of the albendazole-ivermectin combination in lambs parasitized with resistant nematodes. Vet. Parasitol. 155, 249-256.

Fuhr, U., 2007. What is the true risk of a pharmacokinetic drug-drug interaction? Eur. J. Clin. Pharmacol. 63, 897-899.

Gasbarre, L.C., Smith, L.L., Lichtenfels, J.R., Pilitt, P.A., 2009a. The identification of cattle nematode parasites resistant to multiple classes of anthelmintics in a commercial cattle population in the US. Vet. Parasitol. 166, 281-285.

Gasbarre, L.C., Smith, L.L., Hoberg, E., Pilitt, P.A., 2009b. Further characterization of a cattle nematode population with demonstrated resistance to current anthelmintics. Vet. Parasitol. 166, 275-280.

Harrigan, P.R., Hogg, R.S., Dong, W.W.Y., Yip, B., Wynhoven, B., Woodward, J., Brumme, C.J., Brumme, Z.L., Mo, T., Alexander, C.S., Montaner, J.S.G., 2005. Predictors of HIV drug-resistance mutations in a large antiretroviral-naive cohort initiating triple antiretroviral therapy. J. Inf. Dis. 191, 339-347.

Hastings, I.M., 2011. How artemisinin-containing combination therapies slow the spread of antimalarial drug resistance. Trends Parasitol. 27, 167-172.

Hennessy, D.R., Bauer, C., Boray, J.C., Conder, G., Daugschies, A., Johansen, M.-V., Maddox-Hyttel, C., Roepstorff, A., 2006. World Association for the Advancement of Veterinary Parasitology (W.A.A.V.P.) second edition of guidelines for evaluating the efficacy of anthelmintics in swine. Vet. Parasitol. 141, 138-149.

Hosking, B.C., 2010. The control of gastro-intestinal nematodes in sheep with the amino-acetonitrile derivative, monepantel with a particular focus on Australia and New Zealand. Ph.D. Thesis. Ghent University, Belgium. ISBN: 978-9058-642-004.

Jabbar, A., Iqbal, Z., Kerboeuf, D., Muhammad, G., Khan, M.N., Afaq, M., 2006. Anthelmintic resistance: the state of play revisited. Life Sci. 79 , 2413-2431.

Jacobs, D.E., Arakawa, A., Courtney, C.H., Gemmell, M.A., McCall, J.W. Myers, G.H., Vanparijs, O., 1994. World Association for the Advancement of Veterinary Parasitology (W.A.A.V.P.) guidelines for evaluating the efficacy of anthelmintics for dogs and cats. Vet. Parasitol. 52, 179-202.

Kaplan, R.M., 2004. Drug resistance in nematodes of veterinary importance: a status report. Trends Parasitol. 20, 477-481.

Kaplan, R.M., Vidyashankar, A.M., 2012. An inconvenient truth: global worming and anthelmintic resistance. Vet. Parasitol. 186, 70-78.

Kenyon, F., Greer, A.W., Coles, G.C., Cringoli, G., Papadopoulos, E., Cabaret, J., Berrag, B., Varady, M., van Wyk, J.A., Thomas, E., Vercruysse, J. Jackson, F., 2009. The role of targeted selective treatments in the 
development of refugia-based approaches to the control of gastrointestinal nematodes of small ruminants. Vet. Parasitol. 164, 3-11.

Lane, D., 2006. Designer combination therapy for cancer. Nat. Biotechnol. $24,163-164$

Lanusse, C., 2010. Anthelmintic therapy in ruminant species: understanding of the host-drug-parasite interaction. Anti-infect. Agents Med. Chem. 9, 130-138.

Lawrence, K.E., Leathwick, D.M., Rhodes, A.P., Jackson, R., Heuer, C., Pomroy, W.E., West, D.M., Waghorn, T.S., Moffat, J.R., 2007. Management of gastrointestinal nematode parasites on sheep farms in New Zealand. N. Z. Vet. J. 55, 228-234

Leathwick, D.M., 2012. Modelling the benefits of releasing a new class of anthelmintic in combination. Vet. Parasitol. 186, 93-100.

Leathwick, D.M., Hosking, B.C., Bisset, S.A., McKay, C.H., 2009. Managing anthelmintic resistance: is it feasible in New Zealand to delay the emergence of resistance to a new anthelmintic class? N. Z. Vet. J. 57, 181-192.

Leathwick, D.M., Waghorn, T.S., Miller, C.M., Candy, P.M., Oliver, A.M., 2012. Managing anthelmintic resistance - use of a combination anthelmintic and leaving some lambs untreated to slow the development of resistance to ivermectin. Vet. Parasitol. 187, 285-294.

LeJambre, L.F., 1978. Anthelmintic resistance in gastrointestinal nematodes of sheep. In: Donald, A.D., Southcott, W.H., Dineen, J.K. (Eds.), The Epidemiology and Control of Gastrointestinal Parasites of Sheep in Australia. CSIRO, Melbourne, Australia, pp. 109-120

Little, P.R., Hodge, A., Watson, T.G., Seed, J.A., Maeder, S.J., 2010. Field efficacy and safety of an oral formulation of the novel combination anthelmintic, derquantel-abamectin, in sheep in New Zealand. N. Z. Vet. J. 58, 121-129.

Little, P.R., Hodge, A., Maeder, S.J., Wirtherle, N.C., Nicholas, D.R., Cox, G.C., Conder, G.A., 2011. Efficacy of a combined oral formulation of derquantel-abamectin against the adult and larval stages of nematodes in sheep, including anthelmintic-resistant strains. Vet. Parasitol. $181,180-193$.

Love, S.C.J., Neilson, F.J.A., Biddle, A.J., McKinnon, R., 2003. Moxidectinresistant Haemonchus contortus in sheep in northern New South Wales. Aust. Vet. J. 81, 359-360.

Mani, G.S., 1985. Evolution of resistance in the presence of two insecticides. Genetics 109, 761-783.

Martin, P.J., LeJambre, L.F., Claxton, J.H., 1981. The impact of refugia on the development of thiabendazole resistance in Haemonchus contortus. Int. J. Parasitol. 11, 35-41.

McKenna, P.B., 1990. The use of benzimidazole-levamisole mixtures for the control and prevention of anthelmintic resistance in sheep nematodes: an assessment of their likely effects. N. Z. Vet. J. 38, 45-49.

Michel, J.F., 1985. Strategies for the use of anthelmintics in livestock and their implications for the development of drug resistance. Parasitology 90, 621-628.

Miles, D., von Minckwitz, G., Seidman, A.D., 2002. Combination versus sequential single-agent therapy in metastatic breast cancer. Oncologist 7 (Suppl. 6), 13-19.

Molento, M.B., Nielsen, M.K., Kaplan, R.M., 2012. Resistance to avermectin/milbemycin anthelmintics in equine cyathostomins - current situation. Vet. Parasitol. 185, 16-24.

Morgan, E.R., Hosking, B.C., Burston, S., Carder, K.M., Hyslop, A.C., Pritchard, L.J., Whitmarsh, A.K., Coles, G.C., 2012. A survey of helminth control practices on sheep farms in Great Britain and Ireland. Vet. J. 192, 390-397.

Mottier, L., Prichard, R.K., 2008. Genetic analysis of a relationship between macrocyclic lactone and benzimidazole anthelmintic selection on Haemonchus contortus. Pharmacogenet. Genomics 18, 129-140.

Reinemeyer, C.R., 2012. Anthelmintic resistance in non-strongylid parasites of horses. Vet. Parasitol. 185, 9-15.

Sargison, N.D., Jackson, F., Bartley, D.J., Wilson, D.J., Stenhouse, L.J., Penny, C.D., 2007. Observations on the emergence of multiple anthelmintic resistance in sheep flocks in the south-east of Scotland. Vet. Parasitol. $145,65-76$.

Smith, G., 1990. A mathematical model for the evolution of anthelmintic resistance in a direct life cycle nematode parasite. Int. J. Parasitol. 20, 913-921.

Suarez, G., Alvarez, L.I., Castells, D., Moreno, L., Faggiolino, P., Lanusse, C.E., 2009. Pharmacological evaluation of a combined albendazole, ivermectin and levamisole formulation in lambs. In: Proc. World Association for the Advancement of Veterinary Parasitology, Calgary, Canada, pp. 172-173 (Abstract only).

Sutherland, I.A., Damsteegt, A., Miller, C.M., Leathwick, D.M., 2008. Multiple species of nematodes resistant to ivermectin and a benzimidazole-levamisole combination on a sheep farm in New Zealand. N. Z. Vet. J. 56, 67-70.
Sutherland, I.A., Leathwick, D.M., 2011. Anthelmintic resistance in nematode parasites of cattle: a global issue? Trends Parasitol. 27, 176-181.

Taylor, M.A., Hunt, K.R., 1988. Field observations on the control of ovine parasitic gastroenteritis in south-east England. Vet. Rec. 123, 241-245.

Taylor, M.A., Hunt, K.R., 1989. Anthelmintic drug resistance in the UK. Vet. Rec. 125, 143-147.

van Wyk, J.A., Malan, F.S., Randles, J.L., 1997. How long before resistance makes it impossible to control some field strains of Haemonchus contortus in South Africa with any of the modern anthelmintics? Vet. Parasitol. 70, 111-122.

van Wyk, J.A., 2001. Refugia - overlooked as perhaps the most potent factor concerning the development of anthelmintic resistance. Onderstepoort J. Vet. Res. 68, 55-67.

Vercruysse, J., Holdsworth, P., Letonja, T., Barth, D., Conder, G., Hamamoto, K., Okano, K., 2001. International harmonisation of anthelmintic efficacy guidelines. Vet. Parasitol. 96, 171-193.

Vercruysse, J., Holdsworth, P., Letonja, T., Conder, G., Hamamoto, K., Okano, K., Rebhein, S., 2002. International harmonisation of anthelmintic efficacy guidelines (part 2). Vet. Parasitol. 103, 277-297.

Vercruysse, J., Rew, R.S., 2002. General efficacy of the macrocyclic lactones to control parasites of cattle. In: Vercruysse, J., Rew, R.S. (Eds.), Macrocyclic Lactones in Antiparasitic Therapy. Cabi Intl., Wallingford, UK, pp. 185-222.

Waghorn, T.S., Leathwick, D.M., Rhodes, A.P., Jackson, R., Pomroy, W.E., West, D.M., Moffat, J.R., 2006. Prevalence of anthelmintic resistance on 62 beef cattle farms in the North Island of New Zealand. N. Z. Vet. J. $54,278-282$

White, N.J., 1999. Delaying antimalarial drug resistance with combination chemotherapy. Parassitologia 41, 301-308.

Wood, I.B., Amaral, N.K., Bairden, K., Duncan, J.L., Kassai, T., Malone Jr., J.B., Pankavich, J.A., Reinecke, R.K., Slocombe, O., Taylor, S.M., Vercruysse, J., 1995. World Association for the Advancement of Veterinary Parasitology (W.A.A.V.P.) second edition of guidelines for evaluating the efficacy of anthelmintics in ruminants (bovine, ovine, caprine). Vet. Parasitol. 58, 181-213.

Wood, R.J., Mani, G.S., 1981. The effective dominance of resistance genes in relation to the evolution of resistance. Pest. Sci. 12, 573-581.

World Health Organization, 2009. Treatment of Tuberculosis: Guidelines for National Programmes. WHO/CDC/TB/2009.420. WHO, Geneva, Switzerland.

Wrigley, J., McArthur, M., McKenna, P.B., Mariadass, B., 2006. Resistance to a triple combination of broad-spectrum anthelmintics in naturally acquired Ostertagia circumcincta infections in sheep. N. Z. Vet. J. 54, 47-49.

Yazwinski, T.A., Chapman, H.D., Davis, T.J., Letonja, R.B., Pote, L., Maes, L., Vercruysse, J., Jacobs, D.E., 2003. World Association for the Advancement of Veterinary Parasitology (W.A.A.V.P.) guidelines for evaluating the efficacy of anthelmintics in chickens and turkeys. Vet. Parasitol. $116,159-173$

\section{Glossary}

Adequate infection: Natural or induced infection level defined in the study protocol that will allow the evaluation of the therapeutic effectiveness of the drug or product when comparing parasitological parameters (e.g., number of parasites) in medicated and control animals.

Anthelmintic combination product: A pre-formulated single dosage form of two or more constituent anthelmintic actives.

Anthelmintic resistance: The ability of parasites to survive doses of drugs that would normally kill parasites of the same species and stage. It is inherited and selected for because the survivors of drug treatments pass genes for resistance on to their offspring. These genes are initially rare in the population or arise as rare mutations, but as selection continues, the proportion of resistance genes in the population increases as does the proportion of resistant parasites.

Claim: A parasite species or genus (adult and/or larvae) listed on the labeling with proven susceptibility ( $90 \%$ or better effectiveness) to an anthelmintic product.

Controlled study: A study to determine the effectiveness of a drug or product using two groups of adequately infected experimental animals, viz. the treated and control groups. After a suitable period of time after treatment the animals are necropsied, and the parasites enumerated and identified. The percentage efficacy of the drug or product is calculated: $100 \times$ [(geometric mean of parasites in control group) - (geometric mean of parasites in treated group)] divided by (geometric mean of parasites in control group). It should be noted 
that the use of arithmetic rather than geometric means for this purpose remains under investigation, and this method for calculation of efficacy may change in the future.

Dose confirmation study: In vivo study to confirm the effectiveness of a selected drug dose and formulation; may typically be conducted in the field in pens or paddocks.

Dose determination study: In vivo study to determine the most appropriate dose or range of effectiveness of a veterinary drug.

Dose-limiting parasite: A parasite that will be identified during dose determination studies that will identify the dosage of drug at which it shows $90 \%$ effectiveness. Any lower concentration of the drug will show effectiveness below $90 \%$ for the dose-limiting parasite even though it will adequately treat other parasites (90\% or better effectiveness) in the host.

Effectiveness: The degree to which the manufacturers' claims on the labeling have been supported by adequate data, i.e., providing control of at least $90 \%$ on the basis of the calculation of geometric means using pooled data from controlled studies.

Field efficacy study: Larger scale study to determine effectiveness and safety of a veterinary product under actual use conditions.

GCP: Good Clinical Practice: a guideline (from VICH) describes the GCP processes intended to promote the quality and validity of test data. GCP covers the conditions under which studies are planned, performed, monitored, recorded and reported.
Geographical location: A subdivision where the guidelines will be implemented, including but not limited to Australia/New Zealand, the European Union, Japan, the USA, or South America.

(Field) isolate: A collection of a sub-population of helminths for the conduct of drug and product effectiveness tests isolated from the field less than 10 years ago. The helminths are considered representative of current parasitic infections in the field and have been characterized (source, date, location, previous anthelmintic exposure and maintenance procedures).

Multiply drug resistant: The existence of simultaneous resistance to more than one anthelmintic class in a population of a single species of parasitic nematode.

Region: An area within a geographical location defined by climatic conditions, target animal husbandry and parasite resistance prevalence.

Synergism: The interaction of two components of a combination product such that the efficacy is of the components administered together is significantly higher than the sum of the efficacies of the two when administered alone.

VICH: International Cooperation on Harmonisation of Technical Requirements for Registration of Veterinary Medicinal Products.

W.A.A.V.P.: World Association for the Advancement of Veterinary Parasitology. 